\title{
Evaluation of Myo-Inositol as a Potential Biomarker for Depression in Schizophrenia
}

\author{
Joshua Chiappelli',2, Laura M Rowland ${ }^{1,2}$, S Andrea Wijtenburg', Florian Muellerklein', Malle Tagamets', \\ Robert P McMahon', Frank Gaston', Peter Kochunov' and L Elliot Hong*,I \\ 'Department of Psychiatry, Maryland Psychiatric Research Center, University of Maryland School of Medicine, Baltimore, MD, USA
}

\begin{abstract}
Depression is highly prevalent in patients with schizophrenia and is associated with significant clinical consequences, but there is no known biomarker for depression in schizophrenia. One of the putative neurochemical biomarkers for depression in major depressive disorder (MDD) is reduced cerebral concentration of myo-Inositol. We examined whether myo-Inositol levels provide a potential marker for depressive symptoms in schizophrenia similar to that in MDD and are informative regarding causal biological pathways underlying both depression and schizophrenia. We used proton magnetic resonance spectroscopy to examine myo-Inositol levels in the anterior cingulate cortex (ACC) in 59 schizophrenia spectrum disorder (SSD) patients and 69 matched community comparison participants. Participants completed the Maryland Trait and State Depression (MTSD) scale to measure symptoms of depression experienced around time of assessment ('State' subscale) and longitudinally ('Trait' subscale). Myo-Inositol in the ACC was negatively correlated with MTSD-Trait scores in both patients $(\rho=-0.336, p=0.009)$ and community comparison samples $(\rho=-0.328, p=0.006)$. Furthermore, patients with a diagnosis of schizoaffective disorder or a history of at least one major depressive episode had lower levels of myo-Inositol compared with schizophrenia patients without a current or past affective diagnosis $(p=0.012)$. Since reduced brain myo-Inositol is associated with MDD, myo-Inositol may be a biochemical marker of depressive mood symptoms across diagnostic boundaries. If confirmed, this finding may aid investigation of the pathophysiology and therapeutics of depression common between depression, schizophrenia and other psychiatric diagnoses.
\end{abstract}

Neuropsychopharmacology (20I5) 40, 2 I57-2I64; doi:I0.1038/npp.20 I5.57; published online 25 March 2015

\section{INTRODUCTION}

Depression and psychosis are two of the most common psychopathologies and are often co-morbid. Schizophrenia patients with depression have poorer quality of life, higher suicide risk and higher rate of re-hospitalization (Sim et al, 2004; Heilä et al, 1997; Sands and Harrow, 1999). The clinical characteristics of depression in schizophrenia have been extensively studied from a clinical, but not neurobiological perspective (Siris, 2000; Buckley et al, 2009; Bermanzohn et al, 2000). In the largest study of depression of schizophrenia, about 4000 schizophrenia patients were grouped as 'depressed' $v s$ 'non-depressed'. Three-quarters maintained their grouping status after 2-3 years (Conley et al, 2007), suggesting a trait-like property of depression in schizophrenia patients. Importantly, those who changed to nondepressed in 2-3 years improved in $91 \%$ of the functional outcomes assessed; but those who changed to depressed worsened in $94 \%$ of the function outcomes, suggesting that

*Correspondence: Dr LE Hong, Department of Psychiatry, Maryland Psychiatric Research Center, University of Maryland School of Medicine, P.O. Box 21247, Baltimore, MD 21228, USA, Tel: +4I0 402 6828, Fax: +4l0 402 6078, E-mail: ehong@mprc.umaryland.edu

${ }^{2}$ These authors contributed equally to this work.

Received 8 January 2015; revised II February 2015; accepted 16 February 2015; accepted article preview online 27 February 2015 depression may be an important treatment target in schizophrenia. There is currently no known neurobiological marker that tracks the severity of depression in schizophrenia; such a marker may aid treatment development.

Decreased levels of myo-Inositol have been reported in (CSF) and post-mortem frontal cortex of patients with mood disorders (Barkai et al, 1978; Shimon et al, 1997). Glial cells contain a high concentration of myo-Inositol (Brand et al, 1993; Griffin et al, 2002). Myo-Inositol contributes to glial osmoregulatory functioning (Strange, 1992; Fisher et al, 2002), as well as a wide range of other structural and signaling functions. Consistently, proton magnetic resonance imaging $\left({ }^{1} \mathrm{H}-\mathrm{MRS}\right)$ studies of patients with major depressive disorder (MDD) report reduced levels of myo-Insoitol in prefrontal and anterior cingulate cortex (ACC; Frey et al, 1998; Coupland et al, 2005; Chen et al, 2014). Conversely, positive clinical outcome in MDD is associated with increased myo-Inositol levels (Chen et al, 2014; Zheng et al, 2010). These data indicate that reduced myo-Inositol in MDD is primarily associated with depression symptoms rather than being secondary to treatment. Furthermore, several small trials have suggested an antidepressant effect of inositol supplementation (Levine, 1997). In bipolar disorder, some ${ }^{1} \mathrm{H}$-MRS studies suggest frontal myo-Inositol is reduced in depressed periods and normal in euthymic periods, but these findings have been inconsistent and may be confounded by 
the use of mood stabilizers (reviewed in the study by Silverstone et al, 2005).

Previous MRS studies in schizophrenia patients have generally shown no alterations in the concentration of myo-Inositol in cerebral gray matter (as reviewed in the study by Kim et al, 2005; Schwerk et al, 2014). One postmortem study found a lower concentration of myo-Inositol in frontal cortex of patients with schizophrenia (Shimon et al, 1998); this was not replicated (Shapiro et al, 2000), nor were altered levels of inositol found in CSF from schizophrenia patients (Levine et al, 1994). Moreover, inositol supplementation failed to improve total PANSS scores in a small trial of schizophrenia patients (Levine, 1997). However, these studies did not consider whether brain myo-Inositol was related to depression in schizophrenia patients, as would be suggested by studies in MDD. Although the subjective experience of depression is similar between psychotic and mood disorders (Chiappelli et al, 2014b), there have been few studies to examine if the neurobiological correlates of depression also overlap. Therefore, we aimed to examine myo-Inositol in the context of depression in schizophrenia. We previously reported that patients with schizophrenia who have experienced greater frequency of depressive symptoms throughout their adult life had relatively preserved white matter microstructure (Chiappelli et al, 2014a), providing some of the first neurobiological correlates for depression in schizophrenia. Here we extend this line of investigation by examining how brain myo-Inositol is related to current (state) and longitudinal (trait) symptoms of depression in schizophrenia spectrum disorders (SSDs) patients.

\section{MATERIALS AND METHODS}

\section{Participants}

This study included 59 persons with SSD (age range 19-62 years) and 69 age- and sex-matched community controls (age range 18-61 years). Not included in the sample were three participants (two SSD patients and one control) due to head movement and a technical problem with data retrieval, leading to inadequate imaging data, which were excluded prior to any statistical analysis. Diagnoses were confirmed with the Structured Clinical Interview for DSM-IV (SCID) in all participants. Major medical and neurological illnesses, history of head injury with cognitive sequelae, mental retardation, substance dependence within the past 6 months, or current substance abuse (except nicotine) were exclusionary in all participants. SSD patients were recruited from the outpatient clinics at the Maryland Psychiatric Research Center and neighboring mental health clinics. Among the SSD sample, 12 patients had a diagnosis of schizoaffective disorder, and 11 had a history of major depressive episodes. For a subgroup analysis, these 23 patients were defined as 'schizophrenia with a diagnostic mood component' group, in contrast to the remaining group of 36 patients as 'schizophrenia without a diagnostic mood component'. Except for 3 medication-free participants, all SSD patients were on antipsychotic medications, including 14 on clozapine, 4 taking a single typical antipsychotic, 23 taking a single atypical antipsychotic, and 15 taking a combination of antipsychotic medications. Twelve SSD patients in this sample were taking a mood stabilizer at the time of study, including eight on valproic acid, three on lamotrigine, and one on topiramate. Community comparison participants were recruited through media advertisements. Comparison participants had no family history of psychosis in two generations. Most of the comparison participants did not have Axis I diagnoses by SCID, although we did not exclude individuals with a current or past history of a mood disorder, to preserve some variation in depressive symptoms present in the community non-psychotic population. There were two participants in the community sample who had a history of bipolar disorder (one taking a mood stabilizer), and five had a diagnosis of a unipolar depressive disorder (two taking an antidepressant). Participants gave written informed consent approved by the University of Maryland Baltimore IRB.

Clinical data were available for all included participants. Symptoms of depression were measured using the Maryland Trait and State Depression (MTSD) scales, a self-report instrument that is completed in an interview setting. Factor analysis revealed that depression symptoms captured by MTSD in both schizophrenia patients and controls were segregated into two domains: trait and state depression. Trait depression reported by the patients through MTSD was distinct from their negative symptoms (Chiappelli et al, 2014b). The MTSD assesses how frequently symptoms of depression were experienced in the week prior to assessment ('State' subscale) and throughout adult life ('Trait' subscale) (www.mdbrain.org/MTSD_instructions_and_scale.pdf). MTSD scores are reported as average response (scale 0-4) for all items. Overall psychiatric symptoms, including positive psychotic symptoms, were assessed with the item mean of the full 20-item brief psychiatric rating scale (BPRS) and mean of the BPRS psychosis subscale (Overall and Gorham, 1962). The demographics and symptom information are shown in Table 1.

\section{ACC Magnetic Resonance Spectroscopy}

All imaging was acquired using a Siemens $3 \mathrm{~T}$ TRIO MRI (Siemens, Erlangen, Germany) system equipped with a 32 -channel phase array head coil. A $40 \times 30 \times 20 \mathrm{~mm}$ spectroscopic voxel was prescribed on the midsagittal slice and positioned parallel to the genu of the corpus callosum and scalp with the midline of the voxel corresponding to the middle of the genu of the corpus callosum (Figure 1a).

Table I Clinical Information

\begin{tabular}{lcccc}
\hline & SZ $(\boldsymbol{n}=\mathbf{5 9})$ & $\mathbf{C C}(\boldsymbol{n}=\mathbf{6 9})$ & $\mathbf{F}(\mathbf{I}, \mathbf{I} \mathbf{2 6})$ or $\chi^{\mathbf{2}}$ & $\mathbf{p}$ \\
\hline Age & $37.1(12.7)$ & $35.9(13.3)$ & 0.35 & 0.55 \\
Male (\%) & 69.5 & 60.9 & 1.04 & 0.31 \\
Smoker (\%) & 44.1 & 30.4 & 2.54 & 0.11 \\
MTSD-Trait & $1.21(1.08)$ & $0.55(0.62)$ & 18.30 & $<0.001$ \\
MTSD-State & $1.04(0.99)$ & $0.30(0.40)$ & 33.10 & $<0.001$ \\
BPRS & $2.02(0.19)$ & $\mathrm{NA}$ & $\mathrm{NA}$ & $\mathrm{NA}$ \\
BPRS-psychosis & $2.75(1.19)$ & $\mathrm{NA}$ & $\mathrm{NA}$ & $\mathrm{NA}$ \\
\hline
\end{tabular}

Abbreviations: BPRS, brief psychiatric rating scale; CC, community comparison participants; MTSD, Maryland Trait and State Depression scale; SZ, schizophrenia. Except for gender and smoking status, all values represent means with SD in parentheses. 

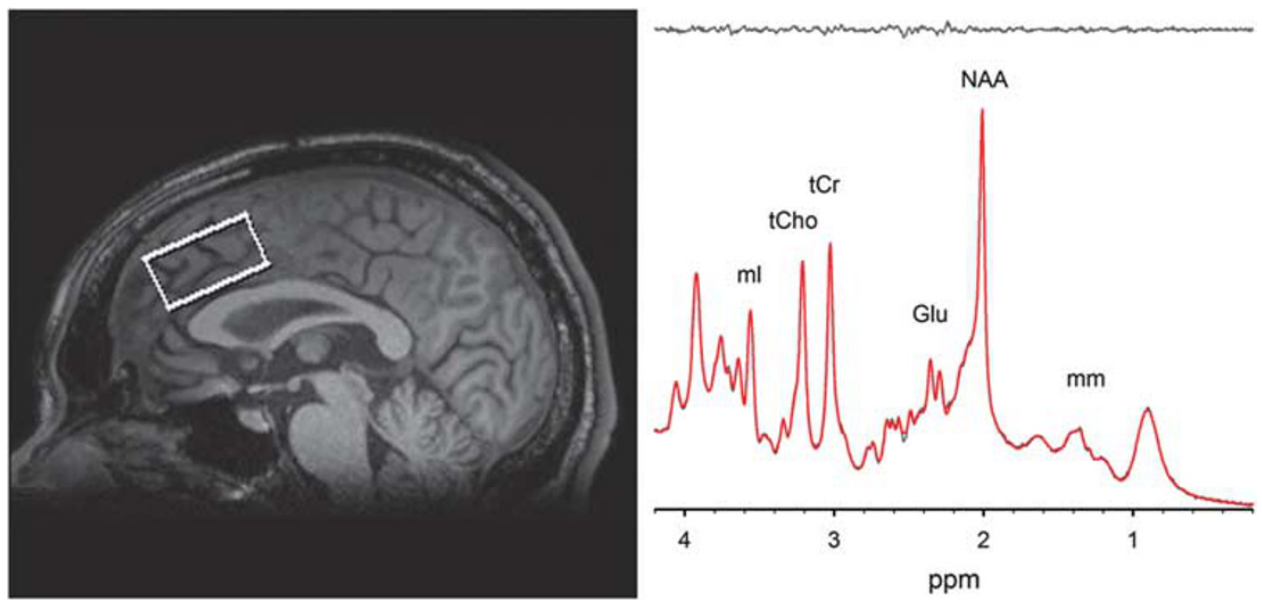

Figure I Voxel placement in anterior cingulate cortex (ACC) and representative spectrum and LCModel metabolite fit (red), with residual shown in gray above.

This medial prefrontal/ACC area was targeted because of the extensive literature supporting its role in both depression and schizophrenia. Imaging studies suggest that the medial prefrontal/ACC is a critical component of neural networks that regulates mood and emotionally influenced behavior in depression (Sacher et al, 2012; Phillips et al, 2003) and also a critical region associated with cognitive and emotional impairment in schizophrenia (Fornito et al, 2009; Reid et al, 2010; Carter et al, 2001; Phillips et al, 2003). Spectra were acquired using very short TE (VTE) phase rotation (PR) STEAM sequence: TR/TM/TE $=2000 / 10 / 6.5-\mathrm{ms}$, $\mathrm{NEX}=128,2.5-\mathrm{kHz}$ spectral width, 2048 complex points, and phases: $\varphi 1=135^{\circ}, \varphi 2=22.5^{\circ}, \varphi 13=112.5^{\circ}, \varphi \mathrm{ADC}=0^{\circ}$ (Wijtenburg and Knight-Scott, 2011; Wijtenburg et al, 2014). A water reference $(\mathrm{NEX}=16)$ was also acquired for phase and eddy current correction as well as quantification. A basis set of 19 metabolites was simulated using the GAVA software package (Soher et al, 2007). The basis set was imported into LCModel (6.3-0I) and used for quantification (Provencher, 1993). Correction for the proportion of the gray matter, white matter, and the CSF within each spectroscopic voxel was performed using SPM8 and in-house Matlab code (Gasparovic et al, 2006). Only metabolites with Cramer Rao lower bounds (CRLB) $<20 \%$ were included in statistical analyses. Spectra with LCModel reported linewidths (LW) $>0.1 \mathrm{~Hz}$ and signal-to-noise ratio (SNR) $<10$ were excluded. Metabolite levels are reported in institutional units (i.u.). Previously, we have shown that the VTE PR-STEAM method produces excellent reproducibility as determined by a test-retest study (Wijtenburg et al, 2014). There were no significant group differences in the quality of the spectra and metabolite fits. The mean ( \pm SD) SNR was $35.6 \pm 7.0$ for controls and $33.1 \pm 8.1$ for patients. The full width half maximum for controls was $0.037 \pm 0.011$ and for patients was $0.038 \pm 0.013$. The CRLB, measures of goodness-of-fit for metabolites, were as follows: myo-Inositol: $3.5 \pm .61 \mathrm{vs}$ $4.0 \pm 1.1$ (control vs patient, respectively; same below); glutamate: $4.7 \pm 1.1$ vs $5.3 \pm 1.4$; glutamate+glutamine (Glx): $5.0 \pm 1.0$ vs $5.5 \pm 1.4 ; \quad \mathrm{N}$-acetylaspartate (NAA) plus $\mathrm{N}$-acetylaspartylglutamate (NAAG) (total NAA): $2.6 \pm 0.7$ vs 2.9 \pm 0.8 ; glutathione (GSH): $7.6 \pm 1.3$ vs $8.5 \pm 2.3$; total choline: $4.6 \pm 1.0$ vs $5.0 \pm 1.6$; total creatine: $2.5 \pm 0.6$ vs $2.8 \pm 0.8$. Proportion of gray matter in the spectroscopic voxel did not significantly differ between patients and controls $(t=0.385, p=0.701)$.

\section{Data Analysis}

Group comparisons for clinical and imaging measures were performed using $t$-tests and one-way ANOVA. Group categorical comparisons used two-sided $\chi^{2}$-tests. The distribution of MTSD data deviated from a normal distribution as determined by Kolmogorov-Smirnov tests, so correlation analyses were performed using Spearman's correlations. An omnibus linear multiple regression was performed to examine the relative contributions of depression, demographic variables, and medication use on myo-Inositol levels. All tests were two-tailed, with significance set to $p<0.05$.

\section{RESULTS}

As reported previously (Chiappelli et al, 2014b), SSD patients had greater levels than controls of both trait (mean \pm SD: $1.21 \pm 1.08$ vs $0.55 \pm 0.62$, respectively, $p<0.001$ ), and state (mean $\pm \mathrm{SD}: 1.04 \pm 0.99$ vs $0.30 \pm 0.40$, respectively, $p<0.001$ ) depression as measured with the MTSD (Table 1). There was no significant difference in ACC myo-Inositol levels between the SSD $(6.84 \pm 0.84)$ and community comparison $(6.94 \pm 0.60)$ samples $(t=0.80, p=0.43$; Figure $2 \mathrm{a})$. However, among the SSD sample, those participants with a diagnostic mood component (schizoaffective disorder or history of one or more major depressive episode) had a significantly lower level of myo-Inositol $(6.50 \pm 0.90)$ than the patient group without a diagnostic mood component $(7.06 \pm 0.74 ; t=2.59, p=0.012$; Figure $2 \mathrm{~b})$. Patients with a diagnostic mood component had significantly higher trait depression (mean \pm SD: $1.71 \pm 1.11$ vs $0.88 \pm 0.95$, respectively, $p=0.003$ ) though not state depression (mean \pm SD: $1.34 \pm 0.94$ vs $0.85 \pm 0.99$, respectively, $p=0.07)$ compared with patients without a diagnostic mood component.

To examine how myo-Inositol levels may track depressive symptom severity, correlations between myo-Inositol and 

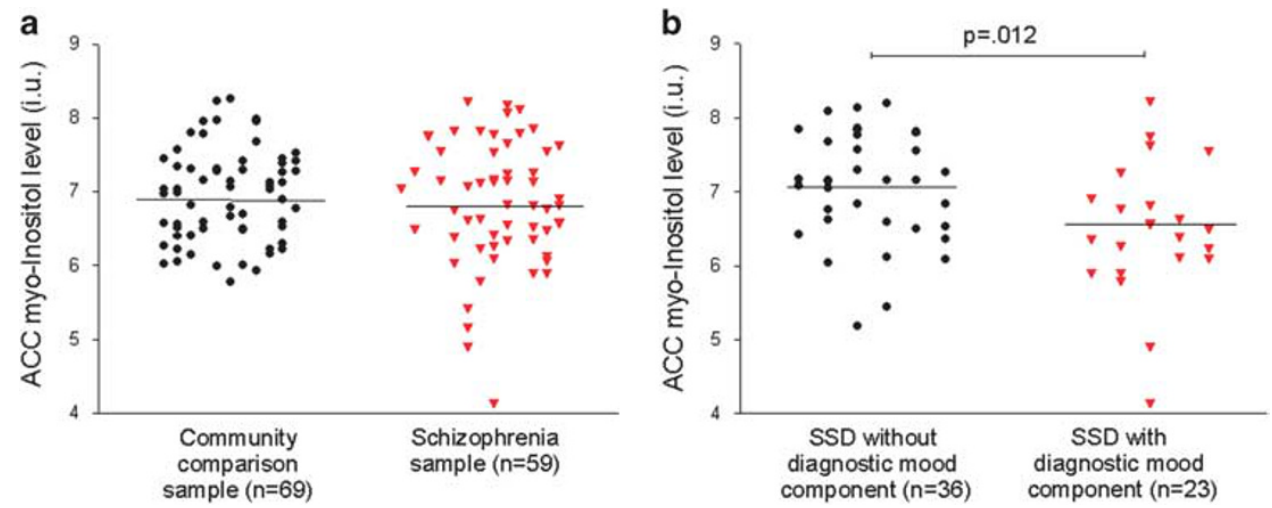

Figure 2 Scatter plots and mean (horizontal lines) myo-Inositol concentrations in anterior cingulate cortex (ACC) compared (a) between community comparison participants and the schizophrenia spectrum disorder (SSD) participants and (b) between SSD with and without a diagnostic mood component (history of schizoaffective disorder or major depressive episode).
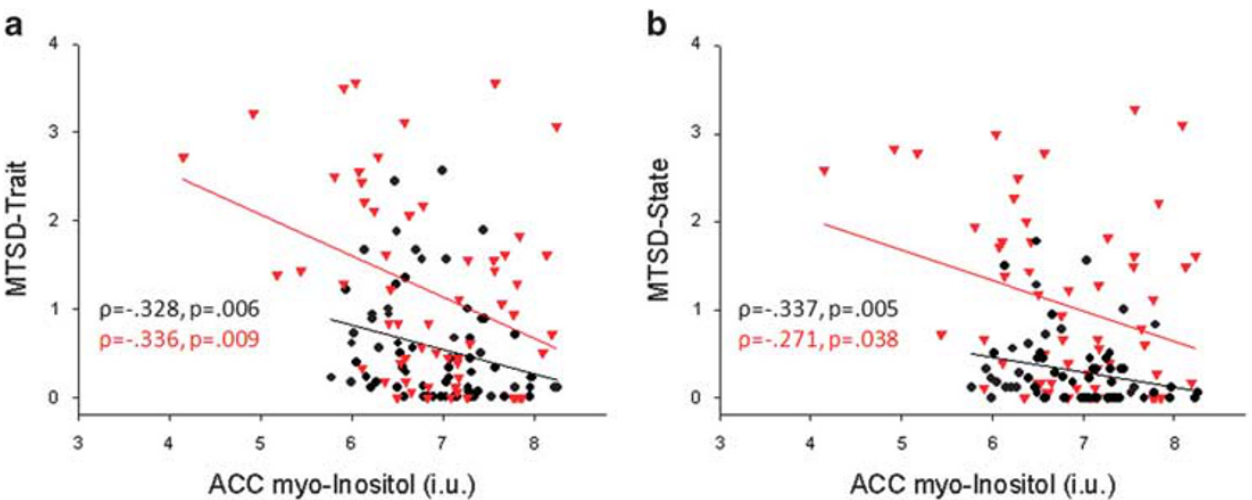

Figure 3 Scatterplots displaying relationship between myo-Inositol levels and Maryland Trait and State Depression (MTSD)-Trait (a) and MTSD-State (b) scores for schizophrenia patients (red triangles) and community comparison sample (black circles). ACC, anterior cingulate cortex.

MTSD scores were examined in each sample independently. In the community comparison sample, myo-Inositol levels were significantly and inversely correlated with both MTSDTrait $(\rho=-0.328, p=0.006)$ and MTSD-State $(\rho=-0.337$, $p=0.005)$ scores. In the SSD, myo-Inositol was also inversely and significantly correlated with MTSD-Trait $(\rho=-0.336$, $p=0.009)$ and MTSD-State $(\rho=-0.271, p=0.038)$ scores. Using Bonferroni correction to control for multiple testing, only trait depression was statistically significant. Therefore, an inverse correlation between ACC myo-Inositol level and trait depression is a replicable finding across the two independent samples with significantly different levels of trait depression, with and without an SSD diagnosis (Figure 3). After removing controls with any history of a mood disorder there was still a trend level, inverse relationship between myo-Inositol levels, and MTSD-State $(\rho=$ $-0.243, p=0.058)$ and MTSD-Trait $(\rho=-0.237, p=0.064)$ in the community comparison sample.

To examine the effects of demographic variables or medication usage on the relationship between depression and myo-Inositol levels, a linear regression analysis was performed using myo-Inositol level as the outcome variable, and diagnosis (SSD versus community control), MTSD-Trait and MTSD-State scores, age, gender, smoking status (current smoker or nonsmoker), antidepressant use (current use or not), and mood stabilizer (current use or not) used as predictor variables. This regression model was significant $(\mathrm{F}(8,119)=4.89, p<0.001)$. MTSD-Trait scores remained a significant negative predictor of myo-Inositol levels $(\beta=-0.261, p=0.035)$, however, MTSD-State scores were not $(\beta=-0.053, p=0.68)$. Although highly correlated with each other, MTSD Trait and State scores had acceptable variance inflation factors (VIF) within this model (2.37 and 2.51 , respectively); a frequently used 'rule of thumb' is that a VIF $>5$ represents unacceptable colinnearity (O’brien, 2007). This model also indicated that age was positively correlated with myo-Inositol levels $(\beta=0.270, p=0.001)$ and that use of a mood stabilizer was associated with lower myoInositol $(\beta=-0.229, p=0.009)$. Diagnosis (SSD vs control) $(\beta=0.064, \quad p=0.52)$ and antidepressant use $(\beta=0.062$, $p=0.51)$ were not significant predictors of myo-Inositol level. A separate linear regression analysis was conducted in the SSD sample alone, entering the same variables above with the exception of diagnosis and MTSD-State scores, but also including chlorpromazine dose equivalent of antipsychotic medication. This model was also significant 
Table 2 Spearman's Correlation Coefficients of MTSD-Trait and State Scores With Other Metabolites Measured With 'H-MRS

\begin{tabular}{lccccc}
\hline & \multicolumn{2}{c}{$\begin{array}{c}\text { Community } \\
\text { comparison participants }\end{array}$} & & \multicolumn{2}{c}{$\begin{array}{c}\text { Schizophrenia } \\
\text { spectrum disorder }\end{array}$} \\
\cline { 2 - 3 } & $\begin{array}{c}\text { MTSD- } \\
\text { Trait }\end{array}$ & $\begin{array}{c}\text { MTSD- } \\
\text { State }\end{array}$ & & $\begin{array}{c}\text { MTSD- } \\
\text { Trait }\end{array}$ & $\begin{array}{c}\text { MTSD- } \\
\text { State }\end{array}$ \\
\hline Glutamate & -0.094 & -0.067 & & 0.058 & -0.003 \\
Glutamate+ & -0.071 & -0.067 & & 0.137 & 0.035 \\
glutamine & & & & & \\
NAA & -0.108 & -0.075 & & -0.168 & -0.232 \\
GSH & 0.070 & -0.030 & & -0.116 & -0.066 \\
Choline & -0.245 & -0.124 & & $-0.388^{\mathrm{a}}$ & -0.277 \\
Creatine & -0.231 & -0.240 & & -0.063 & 0.049 \\
\hline
\end{tabular}

Statistically significant after Bonferroni correction $(p \leqslant 0.002)$

$(\mathrm{F}(7,51)=2.39, \quad p=0.034)$ with no important change in the slope estimating linear association of MTSD-Trait with myo-Inositol $(\beta=-0.285, p=0.037)$, and current antipsychotic medication dosage showed no significantly independent effect on myo-Inositol $(\beta=0.025, p=0.86)$.

There were no significant relationships between myoInositol and BPRS total scores $(r=-0.10, p=0.44)$ or BPRS psychosis subscale $(r=-0.06, p=0.67)$, suggesting that myo-Inositol levels were not significantly associated with psychosis symptoms in general, but were more specifically related to depression. Finally, the current MRS sequence also provides data on levels of the following prominent metabolites: glutamate, Glx, total NAA, glutathione, total creatine, and total choline. The relationship between these metabolites and depression, as a means to test for the specificity of the myo-Inositol finding, was explored (Table 2). Giving the lack of a priori hypotheses, this exploratory analysis employed Bonferroni correction for 24 comparisons (6 MRS measures $\times 2$ groups $\times$ state/trait depression or $p<0.0021)$. Glutamate, Glx, total NAA, glutathione, and total creatine were not significantly associated with depression in either groups (all $p>0.05$ uncorrected). Interestingly, in SSD choline was negatively associated with trait depression $(\rho=-0.388$, $p=0.002$, significant after Bonferroni correction). Choline was also negatively correlated with trait depression in the community comparison sample $(\rho=-0.245, p=0.045)$, though this was not significant. Therefore, among the metabolites measured by this MRS sequence, the relationship of myo-Inositol to depression is relatively specific and replicable across groups.

\section{DISCUSSION}

We found that depressive symptoms in SSD patients were associated with reduced levels of ACC myo-Inositol, a finding consistent with previous studies of patients with MDD. Interestingly, even in our community comparison sample, in which rates of depressive symptoms are overall low, the association between depression and reduced myo-Inositol was observed. This indicates that low levels of myo-Inositol may be a biomarker correlated with depressive symptoms across the current DSM diagnostic boundaries between mood and psychosis diagnoses.

The underlying mechanism linking myo-Inositol to depression in schizophrenia or MDD is unclear. Although neurons exhibit activity-dependent uptake of myo-Inositol (Uldry et al, 2004), myo-Inositol is more concentrated in glial cells than neurons (Brand et al, 1993; Griffin et al, 2002), and may be critical for glial osmoregulatory functioning (Strange, 1992; Fisher et al, 2002). In neuro-inflammatory conditions, elevated myo-Inositol levels are presumed to reflect astrocyte and microglial activation (Chang et al, 2014, Kirov et al, 2013); conversely, reduced myo-Inositol levels have been interpreted as evidence of astrocyte necrosis in neuromyelitis optica (Ciccarelli et al, 2013). Several postmortem studies have found reduced density of glial cells (Ongür et al, 1998; Hamidi et al, 2004) and decreased concentration of protein markers specific to astrocytes in MDD (MiguelHidalgo et al, 2000; Miguel-Hidalgo et al, 2011; MiguelHidalgo et al, 2014), providing a histological basis for interpreting reduced myo-Inositol levels in MDD as evidence of glial dysfunction (Coupland et al, 2005; Chen et al, 2014). Analysis of post-mortem tissue has also implicated reduction in glial numbers in schizophrenia (Rajkowska et al, 2002; Stark et al, 2004), though MRS studies, including this current study, have generally found no evidence of altered levels of myo-Inositol in schizophrenia (Kim et al, 2005; Schwerk et $a l, 2014)$. Stress-induced disruption of glial-neuronal interaction has recently been proposed as a mechanism to explain the cross-diagnostic impact of glial deficits on mood regulation (Edgar and Sibille, 2012), a hypothesis that could potentially be expanded by integrating the osmoregulatory and cell signaling functions of myo-Inositol, and thereby present new opportunities for translational research in depression and schizophrenia.

The inositol pathway has been extensively studied for mechanistic insights into the therapeutic effects of mood stabilizers in bipolar disorder. Preclinical studies have shown that major mood stabilizers such as lithium, valproic acid, and carbamazepine all can reduce myo-Inositol levels (Williams et al, 2002). Data from our samples were consistent with these findings, as current use of mood stabilizer was associated with significantly lower level of myo-Inositol in ACC when diagnosis and other clinical factors were controlled for $(\beta=-0.231, p=0.008)$. However, use of mood stabilizers did not explain the observed association of myoInositol levels with depressive symptoms.

Several studies have found evidence that myo-Inositol level in frontal gray matter increases with age (Chang et al, 1996; Reyngoudt et al, 2012). We replicated this, finding age to be positively correlated with myo-Inositol levels after controlling for other demographic variables $(\beta=0.273, p=0.001)$. This finding was present in community controls $(r=0.374$, $p=0.002)$ but was not significant in SSD patients $(r=0.220$, $p=0.09$ ); however, if patients taking mood stabilizers were excluded, the increase of myo-Inositol with age becomes more evident in patients $(r=0.337, p=0.020)$. Other biomarkers previously described in schizophrenia, such as white matter integrity (Kochunov and Hong, 2014) and cortical thickness (van Haren et al, 2011), are typically shown to be reduced or impaired by disease and also usually decline with age. Thus, in the context of biomarker development, this opposite relationship of a biomarker with 
disease $v s$ age is interesting, although the implication of this observation is currently unknown.

A negative correlation was also found between trait depression and ACC choline levels, but only in the patients. Choline containing compounds measured with MRS (phosphocholine and glycerophosphocholine) are found in about twofold greater concentration in glia than neurons (Urenjak et al, 1993), thus this finding may be consistent with the hypothesis that depression is particularly associated with glial dysfunction. Alternatively, choline levels are sometimes interpreted as a sign of membrane phospholipid turnover, which may be associated with cell proliferation and synaptic remodeling; this has been hypothesized to be the basis for the observation that antidepressant medications and electroconvulsive therapy increase choline levels (Ende et al, 2000; Sonawalla et al, 1999), or prevent stress-induced reductions in choline (Czéh et al, 2001). This post-hoc finding requires replication; and if confirmed, future studies should examine the relationship between myo-Inositol and choline at the level of cellular and particular glial pathways.

A strength of this study was the use of the MTSD, which allows the measurement of depression symptoms over two temporal frames: the current load of symptoms, as well as the longitudinally experienced depressive symptoms reported by the patients. The principal finding of greater levels of depression associated with lower myo-Inositol was stronger for the trait-like experience of depressive symptoms than state depression, suggesting the utility of myo-Inositol as a marker that tracks a more stable, longitudinal aspect of psychopathology, as opposed to a state-dependent measure. Our prior work with this instrument suggested a similar factor structure in symptoms of depression between schizophrenia patients and a community comparison sample, and also found that the MTSD-measured depressive symptoms were distinct from negative symptoms (Chiappelli et al, 2014b). The results here provide convergent validity that the experience of depression is comparable between schizophrenia and affective disorders, in that the neurobiological marker myo-Inositol tracks both in a similar manner. However, as this was a cross-sectional study, we cannot make conclusions regarding causal direction in the relationship between symptoms of depression and myo-Inositol. This finding is intriguing, as it hints at the biology involved in depression; at the same time, the correlations are relatively weak, accounting for about $11 \%$ of the variance in depression measured by the MTSD. This is not surprising because many genetic and psychological factors contribute to vulnerability to depression, with most likely acting independent of or upstream to myo-Inositol and its associated pathways. An additional limitation of this study was the wide range of medications used by participants. Although our analyses did not indicate that use of antidepressant or antipsychotic medications had a significant impact on the relationship between depression and myo-Inositol, it would be of interest to examine this relationship in a sample of medication-free participants. The finding regarding the impact of mood stabilizer use on myo-Inositol level is consistent with preclinical studies, but is based on a small sample of participants taking different types of mood stabilizers, and thus needs confirmation from a larger sample.

To our knowledge, this is the largest MRS study of cerebral myo-Inositol in schizophrenia and depression thus far.
The results indicate the potential use of myo-Inositol as a potential biomarker for depression in schizophrenia, and possibly across disorders; however, extensive research is needed to ascertain whether myo-Inositol can be developed into a clinically useful biomarker with appropriate sensitivity and specificity. Depression is a key factor contributing to the clinical heterogeneity of SSD. Further study of the inositol pathway may improve our understanding of clinical heterogeneity in schizophrenia at the level of neurobiological changes. If treatment targets upstream of myo-Inositol are identified, MRS myo-Inositol might be a useful measure to assess target engagement in preclinical and early stage human studies.

\section{FUNDING AND DISCLOSURE}

Support was received from the National Institutes of Health grants R01MH085646, R01DA027680, R01MH094520, R01MH096263, T32MH067533, P50MH103222, and a NARSAD Young Investigator Award. LEH receives support from the National Institute of Health grants R01MH085646, R01DA027680, P50MH103222, and unrestricted research funding from Mitsubishi, Your Energy Systems LLC, and Pfizer. The remaining authors declare no conflict of interest.

\section{REFERENCES}

Barkai AI, Dunner DL, Gross HA, Mayo P, Fieve RR (1978). Reduced myo-inositol levels in cerebrospinal fluid from patients with affective disorder. Biol Psychiatry 13: 65-72.

Bermanzohn PC, Porto L, Arlow PB, Pollack S, Stronger R, Siris SG (2000). Hierarchical diagnosis in chronic schizophrenia: a clinical study of co-occurring syndromes. Schizophr Bull 26: 517-525.

Brand A, Richter-Landsberg C, Leibfritz D (1993). Multinuclear NMR studies on the energy metabolism of glial and neuronal cells. Dev Neurosci 15: 289-298.

Buckley PF, Miller BJ, Lehrer DS, Castle DJ (2009). Psychiatric comorbidities and schizophrenia. Schizophr Bull 35: 383-402.

Carter CS, MacDonald AW 3rd, Ross LL, Stenger VA (2001). Anterior cingulate cortex activity and impaired self-monitoring of performance in patients with schizophrenia: an event-related fMRI study. Am J Psychiatry 158: 1423-1428.

Chang L, Ernst T, Poland RE, Jenden DJ (1996). In vivo proton magnetic resonance spectroscopy of the normal aging human brain. Life Sci 58: 2049-2056.

Chang L, Jiang C, Cunningham E, Buchthal S, Douet V, Andres M et al (2014). Effects of APOE $\varepsilon 4$, age, and HIV on glial metabolites and cognitive deficits. Neurology 82: 2213-2222.

Chen LP, Dai HY, Dai ZZ, Xu CT, Wu RH (2014). Anterior cingulate cortex and cerebellar hemisphere neurometabolite changes in depression treatment: A $1 \mathrm{H}$ magnetic resonance spectroscopy study. Psychiatry Clin Neurosci 68: 357-364.

Chiappelli J, Kochunov P, DeRiso K, Thangavelu K, Sampath H, Muellerklein F et al (2014a). Testing trait depression as a potential clinical domain in schizophrenia. Schizophr Res 159: 243-248.

Chiappelli J, Nugent KL, Thangavelu K, Searcy K, Hong LE (2014b). Assessment of trait and state aspects of depression in schizophrenia. Schizophr Bull 40: 132-142.

Ciccarelli O, Thomas DL, De Vita E, Wheeler-Kingshott CA, Kachramanoglou C, Kapoor R et al (2013). Low myo-inositol indicating astrocytic damage in a case series of neuromyelitis optica. Ann Neurol 74: 301-305. 
Conley RR, Ascher-Svanum H, Zhu B, Faries DE, Kinon BJ (2007). The burden of depressive symptoms in the long-term treatment of patients with schizophrenia. Schizophr Res 90: 186-197.

Coupland NJ, Ogilvie CJ, Hegadoren KM, Seres P, Hanstock CC, Allen PS (2005). Decreased prefrontal Myo-inositol in major depressive disorder. Biol Psychiatry 57: 1526-1534.

Czéh B, Michaelis T, Watanabe T, Frahm J, de Biurrun G, van Kampen $M$ et al (2001). Stress-induced changes in cerebral metabolites, hippocampal volume, and cell proliferation are prevented by antidepressant treatment with tianeptine. Proc Natl Acad Sci USA 98: 12796-12801.

Edgar N, Sibille E (2012). A putative functional role for oligodendrocytes in mood regulation. Transl Psychiatry 2: e109.

Ende G, Braus DF, Walter S, Weber-Fahr W, Henn FA (2000). The hippocampus in patients treated with electroconvulsive therapy: a proton magnetic resonance spectroscopic imaging study. Arch Gen Psychiatry 57: 937-943.

Fisher SK, Novak JE, Agranoff BW (2002). Inositol and higher inositol phosphates in neural tissues: homeostasis, metabolism and functional significance. $J$ Neurochem 82: 736-754.

Fornito A, Yucel M, Dean B, Wood SJ, Pantelis C (2009). Anatomical abnormalities of the anterior cingulated cortex in schizophrenia: bridging the gap between neuroimaging and neuropathology. Schizophr Bull 35: 973-993.

Frey R, Metzler D, Fischer P, Heiden A, Scharfetter J, Moser E et al (1998). Myo-inositol in depressive and healthy subjects determined by frontal $1 \mathrm{H}$-magnetic resonance spectroscopy at 1.5 tesla. J Psychiatr Res 32: 411-420.

Gasparovic C, Song T, Devier D, Bockholt HJ, Caprihan A, Mullins PG et al (2006). Use of tissue water as a concentration reference for proton spectroscopic imaging. Magn Reson Med 55: 1219-1226.

Griffin JL, Bollard M, Nicholson JK, Bhakoo K (2002). Spectral profiles of cultured neuronal and glial cells derived from HRMAS (1)H NMR spectroscopy. NMR Biomed 15: 375-384.

Hamidi M, Drevets WC, Price JL (2004). Glial reduction in amygdala in major depressive disorder is due to oligodendrocytes. Biol Psychiatry 55: 563-569.

Heilä $\mathrm{H}$, Isometsä ET, Henriksson MM, Heikkinen ME, Marttunen MJ, Lönnqvist JK (1997). Suicide and schizophrenia: a nationwide psychological autopsy study on age- and sex-specific clinical characteristics of 92 suicide victims with schizophrenia. Am J Psychiatry 154: 1235-1242.

Kim H, McGrath BM, Silverstone PH (2005). A review of the possible relevance of inositol and the phosphatidylinositol second messenger system (PI-cycle) to psychiatric disordersfocus on magnetic resonance spectroscopy (MRS) studies. Hum Psychopharmacol 20: 309-326.

Kirov II, Tal A, Babb JS, Herbert J, Gonen O (2013). Serial proton MR spectroscopy of gray and white matter in relapsingremitting MS. Neurology 80: 39-4.

Kochunov P, Hong LE (2014). Neurodevelopmental and neurodegenerative models of schizophrenia: white matter at the center stage. Schizophr Bull 40: 721-728.

Levine J (1997). Controlled trials of inositol in psychiatry. Eur Neuropsychopharmacol 7: 147-155.

Levine J, Goldberger I, Rapaport A, Schwartz M, Schield C, Elizur A et al (1994). CSF inositol in schizophrenia and high-dose inositol treatment of schizophrenia. Eur Neuropsychopharmacol 4: 487-490.

Miguel-Hidalgo JJ, Baucom C, Dilley G, Overholser JC, Meltzer HY, Stockmeier CA et al (2000). Glial fibrillary acidic protein immunoreactivity in the prefrontal cortex distinguishes younger from older adults in major depressive disorder. Biol Psychiatry 48: 861-873.

Miguel-Hidalgo JJ, Overholser JC, Jurjus GJ, Meltzer HY, Dieter L, Konick L et al (2011). Vascular and extravascular immunoreactivity for intercellular adhesion molecule 1 in the orbitofrontal cortex of subjects with major depression: agedependent changes. J Affect Disord 132: 422-431.

Miguel-Hidalgo JJ, Wilson BA, Hussain S, Meshram A, Rajkowska G, Stockmeier CA (2014). Reduced connexin 43 immunolabeling in the orbitofrontal cortex in alcohol dependence and depression. J Psychiatr Res 55: 101-109.

O'brien RM (2007). A caution regarding rules of thumb for variance inflation factors. Quality Quantity 41: 673-690.

Ongür D, Drevets WC, Price JL (1998). Glial reduction in the subgenual prefrontal cortex in mood disorders. Proc Natl Acad Sci USA 95: 13290-13295.

Overall JE, Gorham DR (1962). The brief psychiatric rating scale. Psychol Rep 10: 799-812.

Phillips ML, Drevets WC, Rauch SL, Lane R (2003). Neurobiology of emotion perception II: implications for major psychiatric disorders. Biol Psychiatry 54: 515-528.

Provencher SW (1993). Estimation of metabolite concentrations from localized in vivo proton NMR spectra. Magn Reson Med 30: 672-679.

Rajkowska G, Miguel-Hidalgo JJ, Makkos Z, Meltzer H, Overholser J, Stockmeier C (2002). Layer-specific reductions in GFAP-reactive astroglia in the dorsolateral prefrontal cortex in schizophrenia. Schizophr Res 57: 127-138.

Reid MA, Stoeckel LE, White DM, Avsar KB, Bolding MS, Akella NS et al (2010). Assessments of function and biochemistry of the anterior cingulate cortex in schizophrenia. Biol Psychiatry 68: 625-633.

Reyngoudt H, Claeys T, Vlerick L, Verleden S, Acou M, Deblaere K et al (2012). Age-related differences in metabolites in the posterior cingulate cortex and hippocampus of normal ageing brain: a 1H-MRS study. Eur J Radiol 81: e223-e231.

Sacher J, Neumann J, Fünfstück T, Soliman A, Villringer A, Schroeter ML (2012). Mapping the depressed brain: a metaanalysis of structural and functional alterations in major depressive disorder. J Affect Disord 140: 142-148.

Sands JR, Harrow M (1999). Depression during the longitudinal course of schizophrenia. Schizophr Bull 25: 157-171.

Schwerk A, Alves FD, Pouwels PJ, van Amelsvoort T (2014). Metabolic alterations associated with schizophrenia: a critical evaluation of proton magnetic resonance spectroscopy studies. J Neurochem 128: 1-87.

Shapiro J, Belmaker RH, Biegon A, Seker A, Agam G (2000). Scyllo-inositol in post-mortem brain of bipolar, unipolar and schizophrenic patients. J Neural Transm 107: 603-607.

Shimon H, Agam G, Belmaker RH, Hyde TM, Kleinman JE (1997). Reduced frontal cortex inositol levels in postmortem brain of suicide victims and patients with bipolar disorder. Am J Psychiatry 154: 1148-1150.

Shimon H, Sobolev Y, Davidson M, Haroutunian V, Belmaker RH, Agam G (1998). Inositol levels are decreased in postmortem brain of schizophrenic patients. Biol Psychiatry 44: 428-432.

Silverstone PH, McGrath BM, Kim H (2005). Bipolar disorder and myo-inositol: a review of the magnetic resonance spectroscopy findings. Bipolar Disord 7: 1-10.

Sim K, Mahendran R, Siris SG, Heckers S, Chong SA (2004). Subjective quality of life in first episode schizophrenia spectrum disorders with comorbid depression. Psychiatry Res 129: $141-147$.

Siris SG (2000). Depression in schizophrenia: perspective in the era of 'Atypical' antipsychotic agents. Am J Psychiatry 157: 1379-1389.

Soher BJ, Young K, Bernstein A, Aygula Z, Maudsley AA (2007). GAVA: spectral simulation for in vivo MRS applications. J Magn Reson 185: 291-299.

Sonawalla SB, Renshaw PF, Moore CM, Alpert JE, Nierenberg AA, Rosenbaum JF et al (1999). Compounds containing cytosolic 
choline in the basal ganglia: a potential biological marker of true drug response to fluoxetine. Am J Psychiatry 156: 1638-1640.

Stark AK, Uylings HB, Sanz-Arigita E, Pakkenberg B (2004). Glial cell loss in the anterior cingulate cortex, a subregion of the prefrontal cortex, in subjects with schizophrenia. Am J Psychiatry 161: $882-888$.

Strange K (1992). Regulation of solute and water balance and cell volume in the central nervous system. J Am Soc Nephrol 3: 12-27.

Uldry M, Steiner P, Zurich MG, Béguin P, Hirling H, Dolci W et al (2004). Regulated exocytosis of an $\mathrm{H}+/$ myo-inositol symporter at synapses and growth cones. EMBO J 23: 531-540.

Urenjak J, Williams SR, Gadian DG, Noble M (1993). Proton nuclear magnetic resonance spectroscopy unambiguously identifies different neural cell types. J Neurosci 13: 981-989.

van Haren NE, Schnack HG, Cahn W, van den Heuvel MP, Lepage C, Collins L et al (2011). Changes in cortical thickness during the course of illness in schizophrenia. Arch Gen Psychiatry 68: 871-880.

Wijtenburg SA, Knight-Scott J (2011). Very short echo time improves the precision of glutamate detection at $3 \mathrm{~T}$ in $1 \mathrm{H}$ magnetic resonance spectroscopy. J Magn Reson Imaging 34: 645-652.

Wijtenburg SA, Gaston FE, Spieker EA, Korenic SA, Kochunov P, Hong LE et al (2014). Reproducibility of phase rotation STEAM at 3T: focus on glutathione. Magn Reson Med 72: 603-609.

Williams RS, Cheng L, Mudge AW, Harwood AJ (2002). A common mechanism of action for three mood-stabilizing drugs. Nature 417: 292-295.

Zheng H, Zhang L, Li L, Liu P, Gao J, Liu X et al (2010). Highfrequency rTMS treatment increases left prefrontal myo-inositol in young patients with treatment-resistant depression. Prog Neuropsychopharmacol Biol Psychiatry 34: 1189-1195. 